\title{
Polyunsaturated Fatty Acids and Cardiovascular Disease: Implications for Nutrigenetics
}

\author{
Hooman Allayee ${ }^{a, b}$ Nitzan Roth ${ }^{a}$ Howard N. Hodis ${ }^{a, c}$ \\ ${ }^{a}$ Department of Preventive Medicine, ${ }^{b}$ Institute for Genetic Medicine and 'Atherosclerosis Research Unit, \\ Keck School of Medicine, University of Southern California, Los Angeles, Calif., USA
}

\section{Key Words}

Cardiovascular disease $\cdot$ Genes $\cdot$ Fatty acids .

5 -Lipoxygenase $\cdot$ Leukotriene

\begin{abstract}
Cardiovascular disease (CVD) arises as a result of genetic predisposition in the context of a disease-promoting environment. While several risk factors have been identified for CVD, such as elevated serum lipid levels and hypertension, most of the genes identified thus far do not appear to involve such 'conventional' risk factors. Moreover, the interactions between genes and environment, such as a diet high in certain fats, adds another level of complexity to CVD and renders identification of the underlying genetic factors even more difficult. Polyunsaturated fatty acids (PUFAs), such as the $\omega-$ 6 and $\omega-3$ fatty acids, which have multiple roles in membrane structure, lipid metabolism, blood clotting, blood pressure, and, in particular, inflammation, have been linked to the reduction in CVD. Linoleic $(\omega-6)$ and $\alpha$-linolenic acid $(\omega-3)$ are essential fatty acids that can be converted into long-chain PUFAs, such as arachidonic acid (AA) and eicosapentaenoic acid (EPA)/docosahexaenoic acid (DHA), respectively. These long-chain PUFAs are metabolized by enzymatically catalyzed systems via cyclooxygenases and lipoxygenases. The 5-lipoxygenase (5-LO)/leukotriene (LT) biosynthesis pathway has been biochemically and genetically associated with CVD traits in mice and humans, particularly in the context of dietary AA and EPA/DHA. In this review,
\end{abstract}

we summarize the biochemical metabolism of $\omega-3$ and $\omega-6$ PUFAs, evaluate the evidence for genetic and nutrigenetic contributions of 5-LO pathway genes to CVD, and discuss the potential of future studies that could identify other gene-dietary interactions between PUFAs and CVD traits.

Copyright ๑ 2009 S. Karger AG, Basel

\section{The Atherogenic Process}

The most common cause of CVD, atherosclerosis, is a progressive disease characterized by the accumulation of lipids and fibrous elements in large arteries [1]. It is a complex process of numerous factors and mediators involving the dynamic exchange of signals between resident cells, such as endothelial cells and smooth muscle cells, and infiltrating monocyte/macrophages and lymphocytes. The atherogenic process is initiated by the accumulation of low-density lipoprotein (LDL) particles in the subendothelial layer of the artery wall, where they are oxidized by cell-derived reactive oxygen species (fig. 1, step I). The resulting production of adhesion molecules, chemokines, and growth factors by endothelial cells causes inflammatory cells, comprised predominantly of monocytes, to adhere to the vessel wall and migrate into the subendothelial space. In this microenvironment, the activated monocytes proliferate and differentiate into macrophages, which engulf oxidized LDL particles and subsequently transform into foam cells (fig. 1, step II).

\section{KARGER}

() 2009 S. Karger AG, Basel

Fax +41613061234 E-Mail karger@karger.ch www.karger.com www.karger.com/jnn
Hooman Allayee, $\mathrm{PhD}$

Department of Preventive Medicine, USC Keck School of Medicine

2250 Alcazar Street, CSC 206

Los Angeles, CA 90033 (USA)

Tel. +1 323442 1736, Fax +1 332442 2764, E-Mail hallayee@ usc.edu 


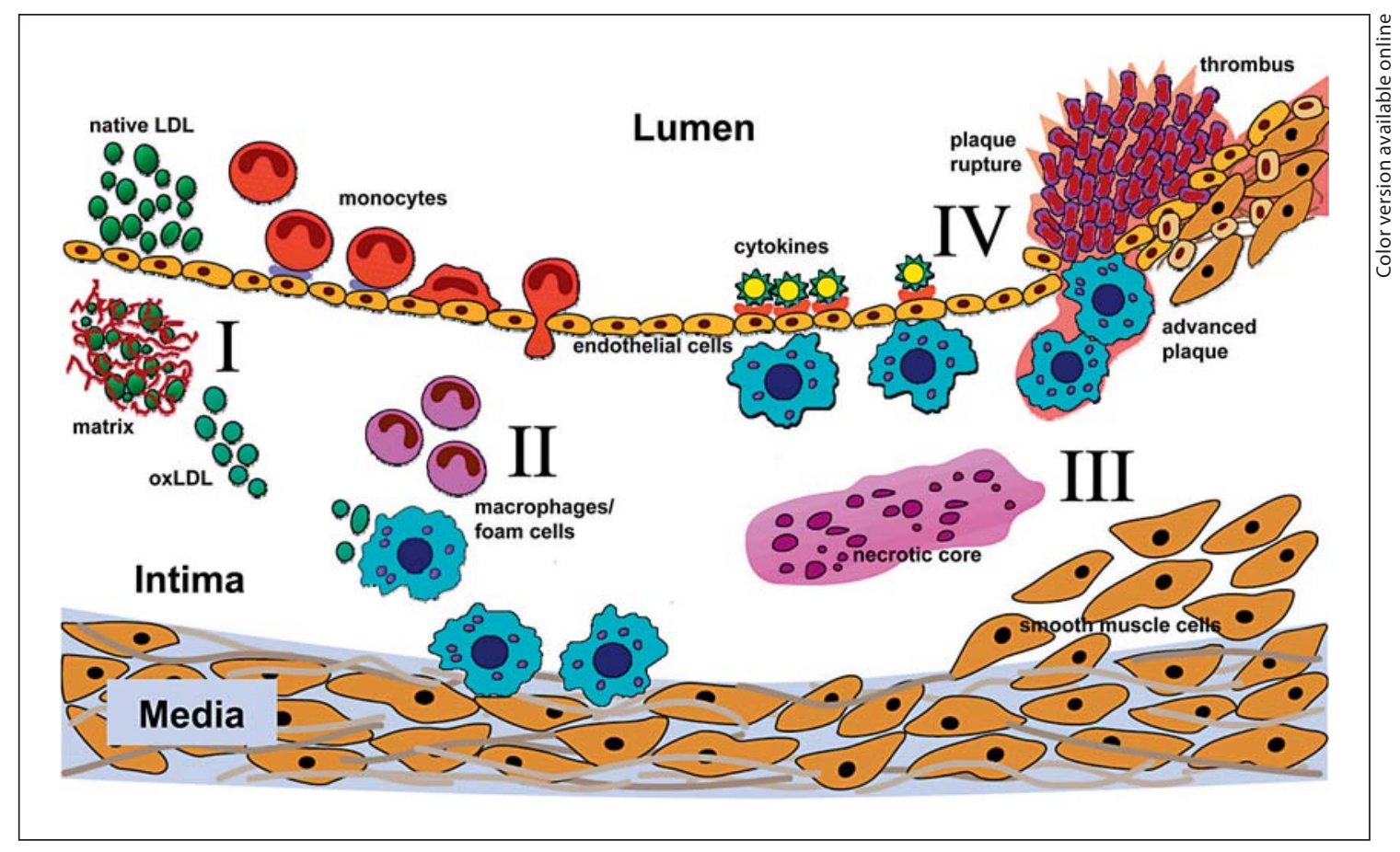

Fig. 1. Steps in the progression of atherosclerosis. Step I: LDL particles accumulate in the sub-endothelial cell layer and become trapped within matrix proteins, where they are oxidized (oxLDL) by cell-derived reactive oxygen species. Step II: Chemotactic and growth factors stimulate monocytes to transmigrate across the endothelial cell layer and differentiate into macrophages. Macrophages engulf the oxLDL, leading to foam cell formation. Step III: Foam cells produce a variety of mediators and eventually undergo apoptosis or necrosis, contributing their contents to a growing core of cellular debris and cholesterol. This process is accompanied by the migration of smooth muscle cells into the intima from the media and the formation of a fibrotic cap. Step IV: As the advanced lesion continues to grow, it becomes increasingly unstable and prone to rupture, which can result in a thrombus or clinical event such as MI or stroke.
Accompanied by a progressive increase in extracellular lipids and intimal smooth muscle cells that have migrated through the media, the resulting 'fatty streaks' develop into advanced lesions as the lipid-laden macrophages undergo apoptosis to form a necrotic core (fig. 1, step III). Proinflammatory cytokines that are also expressed in the lesion cause the smooth muscle cells to proliferate and secrete collagen and other extracellular matrix proteins, resulting in the formation of a fibrotic cap. Such advanced lesions become increasingly complex with calcification, ulceration at the luminal surface, and hemorrhage from small vessels that grow into the lesion from the media, rendering them unstable and prone to rupture (fig. 1, step IV). Ultimately, plaque erosion and rupture can lead to clinical events such as myocardial infarction (MI) or stroke.

Over the past half century, epidemiological studies have revealed numerous risk factors for atherosclerosis [2]. These are, in large part, controlled by traits with ge- netic components, including gender, age, hypertension, hyperlipidemia, obesity, and diabetes. Collectively, these have come to be known as 'conventional' risk factors. The importance of genetics in CVD has been examined in many family and twin studies where heritability estimates for atherosclerosis have been large in most studies, frequently exceeding $50 \%$. While the role of conventional risk factors in CVD is generally accepted, it is also likely that there are genetic contributions to CVD that are independent of known risk factors. For example, certain individuals develop premature heart disease but do not exhibit high cholesterol or hypertension. This may, in part, be due to genetic influences that act at the level of vessel wall metabolism, which lead to variations in cellular function or inflammatory responses among individuals. Recent large genome-wide association studies support this notion since the genes with the strongest signals are not linked to lipid levels and blood pressure [3]. Moreover, these studies also demonstrate the genetic 
complexity underlying CVD since over 10 loci have been identified thus far, which together only explain a fraction of the total genetic variation.

Another level of complexity involves the interactions between genes themselves and with environmental factors, such as stress, a diet high in certain fats, lack of exercise, and smoking. Thus, common forms of CVD result from the interplay between susceptibility genes and lifestyle.

\section{Polyunsaturated Fatty Acids}

Fatty acids are a class of lipid compounds that consist of a hydrocarbon skeleton with a carboxyl group $(-\mathrm{COOH})$ at one end. They are classified as saturated fatty acids (SFAs), monounsaturated fatty acids (MUFAs), or polyunsaturated fatty acids (PUFAs) based on the number of double bonds between atoms in their carbon chain. Saturated fatty acids have no double bonds and are therefore fully saturated with hydrogen atoms, whereas MUFAs and PUFAs have 1 double bond and more than 1 double bond per molecule, respectively. Individual fatty acids can be identified by formal chemical names that indicate the length of the carbon chain and the locations of any double bonds. MUFAs and PUFAs are further classified as $\omega-3, \omega-6$, or $\omega-9$ based on the location of the first double bond from the methyl end of the fatty acid molecule.

Humans are not capable of synthesizing fatty acids with double bonds more than 9 carbons distal from the carboxyl end of the fatty acid [4]. As such, linoleic acid

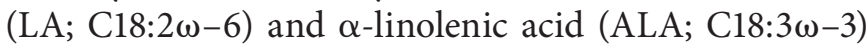
are essential $\omega-6$ and $\omega-3$ PUFAs, respectively, that must be obtained from dietary sources in order to ensure normal physiologic functions [5]. Foods rich in LA include corn and sunflower oils, whereas flaxseed oil is particularly high in ALA. Longer-chain $\omega-6$ and $\omega-3$ PUFAs, such as arachidonic acid (AA; C20:4 $\omega-6)$ and eicosapentaenoic acid (EPA; $\mathrm{C} 20: 5 \omega-3)$, are not strictly 'essential' because they can be synthesized from LA and ALA, respectively, through elongation and desaturation steps catalyzed by the $\Delta 6$ - and $\Delta 5$-desaturase enzymes [6] (fig. 2). In humans, conversion of ALA to EPA occurs slowly, whereby approximately $5 \%$ is converted to EPA, of which $2-5 \%$ is further converted to docosapentaenoic acid [C22:5 $\omega-3$ ] (DHA) [7-9]. Additionally, the large amount of $\omega-6$ fatty acids in the typical Western diet interferes with endogenous synthesis of EPA and DHA from ALA since the $\omega-3$ and $\omega-6$ fatty acids compete for

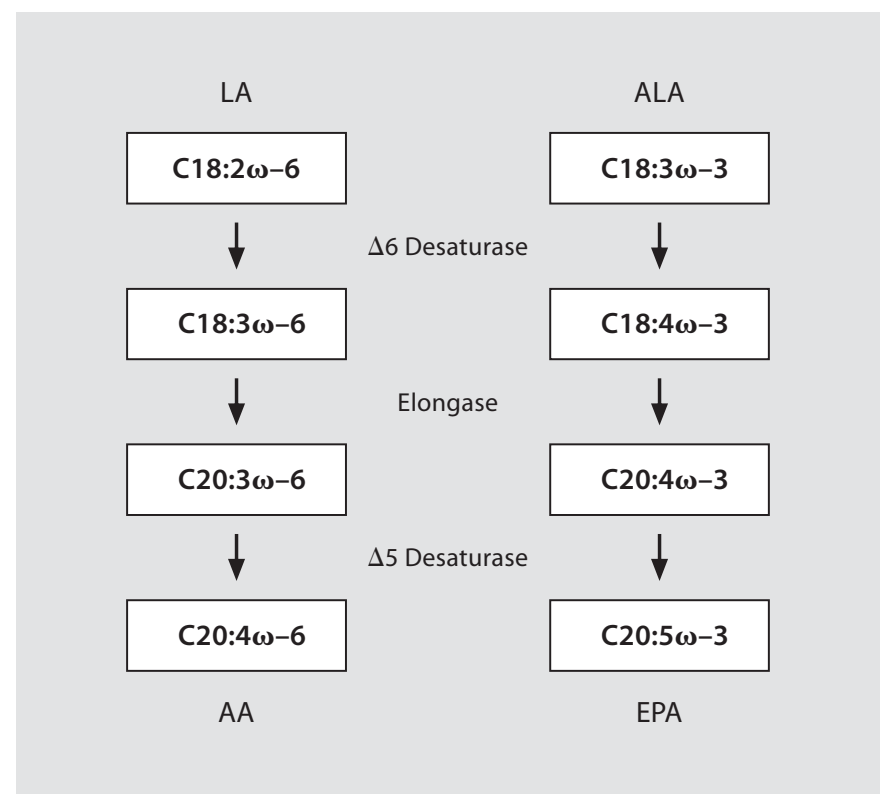

Fig. 2. The enzymes and intermediates of PUFA metabolism. LA and ALA, 2 essential PUFAs that must be obtained from the diet, are desaturated and elongated by the $\Delta 6$ - and $\Delta 5$-desaturase enzymes to generate longer-chained PUFAs, such as AA and EPA.

the same elongase and desaturase enzymes [10]. Moreover, $\omega-6$ PUFAs cannot be converted into $\omega-3$ PUFAs or vice versa in mammalian cells [4]. Thus, dietary recommendations for obtaining sufficient quantities of preformed EPA and DHA are through the consumption of fatty fish or fish oil supplements, both of which are rich sources of such PUFAs.

\section{Inflammation as a Link between PUFAs and CVD}

The cardioprotection conferred by PUFAs, in particular EPA/DHA, has been extensively reviewed elsewhere and is thought to occur through various mechanisms (table 1), including the reduction of serum triglyceride levels, anti-arrhythmic effects, decreasing platelet aggregation, plaque stabilization, and/or reduction of blood pressure [11-14]. Another potentially important link between PUFAs and atherosclerosis may be through inflammation, which can be regulated by the eicosanoids [15-17]. Importantly, the effects of eicosanoids can be driven, in large part, by competition between AA and EPA as substrates for enzymes that catalyze release of the fatty acids from cell membranes or their conversion into a variety of metabolites. In response to a calcium stimulus, cytosolic 
Fig. 3. Generation of eicosanoids from AA and EPA. Metabolism of AA and EPA by the COX and 5-LO pathways leads to the 2,4- or 3,5-series prostanoids and LTs, respectively. The 2,4-series eicosanoids produced from AA promote inflammatory and prothrombotic effects, whereas the corresponding 3,5-series eicosanoids produced from EPA have either the opposite effects or possess much lower levels of biological activity.
AA

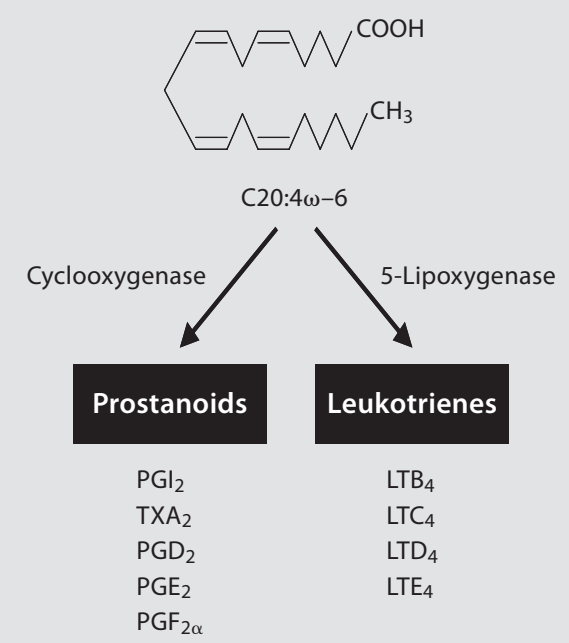

EPA
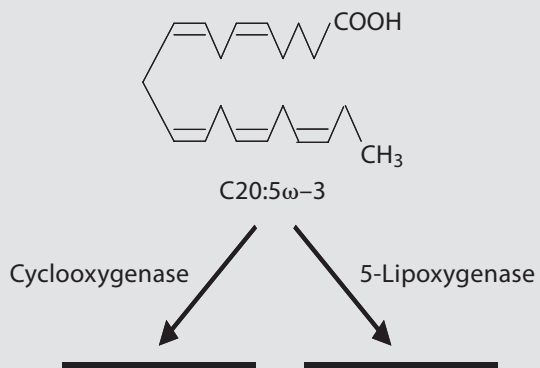

Prostanoids

Leukotrienes

$\mathrm{PGI}_{3}$

$\mathrm{TXA}_{3}$

$\mathrm{PGD}_{3}$

$\mathrm{PGE}_{3}$

$\mathrm{PGF}_{3 \alpha}$
$\mathrm{LTB}_{5}$

$\mathrm{LTC}_{5}$

$\mathrm{LTD}_{5}$

$\mathrm{LTE}_{5}$
Table 1. Cardioprotective effects of EPA/DHA on CVD risk factors

\begin{tabular}{ll}
\hline Effect & Proposed mechanism \\
\hline $\begin{array}{l}\text { Lowered serum } \\
\text { triglycerides }\end{array}$ & $\begin{array}{l}\text { reduction in hepatic triglyceride production and } \\
\text { lipoprotein assembly }\end{array}$ \\
\hline $\begin{array}{l}\text { Anti- } \\
\text { arrhythmic }\end{array}$ & $\begin{array}{l}\text { modulation of electrophysiological properties of } \\
\text { cardiac myocytes }\end{array}$ \\
\hline $\begin{array}{l}\text { Lowered blood } \\
\text { pressure }\end{array}$ & $\begin{array}{l}\text { improved endothelial cell function, } \\
\text { vascular relaxation, and arterial compliance }\end{array}$ \\
\hline $\begin{array}{l}\text { Decreased plate- } \\
\text { let aggregation }\end{array}$ & $\begin{array}{l}\text { reduction in prothrombotic prostanoids } \\
\text { through competition with AA }\end{array}$ \\
\hline $\begin{array}{l}\text { Decreased } \\
\text { inflammation }\end{array}$ & $\begin{array}{l}\text { reduction in 4-series leukotriene production } \\
\text { and signaling through competition with AA and } \\
\text { leukotriene receptor antagonism, respectively }\end{array}$ \\
\hline
\end{tabular}

phospholipase $\mathrm{A}_{2}$ releases membrane bound $\mathrm{AA}$, which then becomes available for eicosanoid synthesis [18]. Eicosanoids can be formed from AA through a variety of enzymatic reactions involving cyclooxygenases (COX) and 5-lipoxygenase (5-LO). The COX-dependent metabolism of AA yields the 2-series prostanoids while the 5LO pathway yields the 4-series leukotrienes (LTs) (fig. 3; table 2), the latter having long been implicated in other allergic inflammatory diseases, such as asthma [19]. 5$\mathrm{LO}$ is the rate-limiting enzyme in LT biosynthesis and is expressed primarily in leukocytes, particularly monocytes and macrophages. Once released, free AA is presented by $5-\mathrm{LO}$ activating protein (FLAP) to $5-\mathrm{LO}$, which incorporates molecular oxygen into AA to generate $\mathrm{LTA}_{4}$. $\mathrm{LTA}_{4}$ can then be converted to $\mathrm{LTB}_{4}$ via $\mathrm{LTA}_{4}$ epoxide hydrolase (LTA4H) or shunted into the cysteinyl LT pathway and converted to $\mathrm{LTC}_{4}$ through the conjugation of glutathione by LTC4 synthase (LTC4S). Importantly, the 2,4-series eicosanoids are proinflammatory and possess many atherogenic properties $[15,20,21]$. For example, thromboxane $\mathrm{A}_{2}$, a major COX product synthesized in platelets, promotes platelet aggregation [22]. By comparison, $\mathrm{LTB}_{4}$ is a potent chemotactic factor for circulating monocytes and neutrophils, whereas $\mathrm{LTC}_{4}, \mathrm{LTD}_{4}$ and $\mathrm{LTE}_{4}$ are the slow-reacting substances of anaphylaxis and mediate bronchoconstriction and smooth muscle contraction [19].

EPA and DHA consumption results in dose-dependent increased incorporation of these fatty acids into cellular phospholipids. Whereas AA-derived eicosanoids are potent mediators of inflammation, the 3,5-series eicosanoids derived from EPA/DHA have anti-inflammatory and anti-atherogenic effects (fig. 2) [12, 16, 23]. The EPAderived prostanoids (i.e. $\mathrm{TXA}_{3}$ ) have little biological activity compared with their corresponding AA-derived counterparts. Similarly, EPA-derived LTs are also biologically less active than their AA-derived counterparts with $\mathrm{LTB}_{5}$ an order of magnitude less active than AA-derived $\mathrm{LTB}_{4}$ [24]. The 5-series LTs also compete with the more 
Table 2. Major genes of the LT biosynthetic pathway

\begin{tabular}{|c|c|}
\hline Gene and symbol & Function \\
\hline Cytosolic phospholipase A2 (cPLA2) & releases AA from cell membranes \\
\hline 5-Lipoxygenase (5-LO) & incorporates oxygen into AA and forms $\mathrm{LTA}_{4}$ \\
\hline 5-Lipoxygenase-activating protein (FLAP) & presents AA to and activates 5 - $\mathrm{LO}$ \\
\hline $\mathrm{LTA}_{4}$ epoxide hydrolase (LTA4H) & converts $\mathrm{LTA}_{4}$ to $\mathrm{LTB}_{4}$ \\
\hline $\mathrm{LTB}_{4}$ omega hydroxylase (LTB4H) & catalyzes the breakdown of $\mathrm{LTB}_{4}$ \\
\hline $\mathrm{LTC}_{4}$ synthase (LTC4S) & converts $\mathrm{LTA}_{4}$ to the first cysteinyl LT, $\mathrm{LTC}_{4}$ \\
\hline $\mathrm{LTB}_{4}$ receptor 1 (LTB4R) & receptor for $\mathrm{LTB}_{4}$ \\
\hline $\mathrm{LTB}_{4}$ receptor 2 (LTB4R2) & a second receptor for $\mathrm{LTB}_{4}$ \\
\hline Cysteinyl leukotriene receptor 1 (CysLTR1) & receptor for cysteinyl LTs, such as $\mathrm{LTD}_{4}$ \\
\hline Cysteinyl leukotriene receptor 2 (CysLTR2) & a second receptor for the cysteinyl LTs \\
\hline
\end{tabular}

active 4-series LTs for their respective receptors (LTB4R and LTB4R2) for $\mathrm{LTB}_{4}$ and type 1 and type 2 cysteinyl LT receptors (CysLTR1 and CysLTR2) for $\mathrm{LTC}_{4}, \mathrm{LTD}_{4}$ and $\mathrm{LTE}_{4}$, which further decreases the inflammatory activity of the 4-series LTs. Thus, the 2,4-series eicosanoids produced from AA promote inflammatory and prothrombotic effects, whereas the corresponding 3,5-series eicosanoids produced from EPA have either the opposite effects or possess much lower levels of biological activity. Since the typical Western diet is rich in LA (and AA) and relatively poor in EPA/DHA, it has been proposed that lowering the dietary $\omega-6 / \omega-3$ ratio would provide an efficient means by which to reduce risk of CVD and other inflammatory conditions [5].

\section{Contribution of LT Pathway Genes to CVD}

The 5-LO pathway has recently garnered a great deal of attention for its potential role in CVD-related traits. This stems from a series of biochemical, genetic, and pharmacological studies in mice and humans over the last several years, which collectively have provided relatively strong evidence for the proatherogenic role of LTs $[20,25,26]$. In an initial study with inbred mouse strains, an atherosclerosis susceptibility locus was mapped to a region of mouse chromosome 6 harboring several potential candidate genes for CVD traits, including that encoding the 5-LO enzyme [27]. In a subsequent study, it was reported that 5-LO-deficient mice on a genetically hyperlipidemic background had dramatically reduced aortic lesion formation despite cholesterol levels in excess of 500 $\mathrm{mg} / \mathrm{dl}$ [28]. These studies were amongst the first to directly implicate a role for 5-LO in atherosclerosis. Subsequent reports have also suggested the involvement of 5-
LO in atherosclerosis-related phenotypes as well [29, 30], but this has not been consistently observed across all studies [31]. More recently, 5-LO has been implicated in metabolic traits, such as adiposity, bone density, and pancreatic dysfunction $[32,33]$. Biochemical evidence in human coronary and carotid plaques also provides evidence that the 5-LO pathway promotes proatherogenic processes. For example, several studies have shown that 5-LO, FLAP, and LTA4H are abundantly expressed in arterial walls of CVD patients, with 5-LO having markedly increased expression in advanced lesions and localizing to inflammatory cells, such as macrophages and dendritic cells [34-36]. More recently, 5-LO gene expression in peripheral blood cells was positively correlated with the extent of coronary atherosclerosis in CVD patients [37]. Thus, 5-LO pathway genes and LTs could potentially contribute to lesion progression in humans, from its initial stages up to the development of complex plaques that are prone to rupture and cause an MI.

In parallel, genetic studies in humans add to the evidence that the 5-LO pathway contributes to the risk of CVD. For example, in a cohort study of healthy individuals, certain alleles of a 5-LO promoter repeat polymorphism, consisting of a variable number of Sp1 transcription factor binding sites, were associated with significantly increased carotid intima-media thickness and plasma biomarkers of inflammation [38]. An interesting extension of these analyses was that high dietary AA exacerbated the atherogenic effect of the variant alleles, whereas high dietary EPA/DHA blunted this effect [38], thereby providing a potential molecular mechanism for the observed association through gene-dietary interactions. In a subsequent study with a Costa Rican sample, the variant 5-LO promoter alleles did not show an association with a clinical phenotype, namely $\mathrm{MI}$ 
[39]. However, the variant alleles did exhibit a nutrigenetic interaction with AA levels where individuals carrying 1 or 2 copies of the shorter variant repeats had an elevated risk of MI in the high dietary AA group compared to homozygotes for the common allele in the low AA group. By contrast, the shorter variants were protective in the context of low dietary AA [39]. It is noteworthy that, without knowledge of the dietary status of subjects, the association of these alleles and MI could be neutralized in an unstratified analysis that considered all individuals together since the gene-diet interaction between the variant 5-LO promoter repeats and MI risk went in opposite directions. Although speculative, such a notion may explain why an association with the promoter repeats and MI has not been detected in other populations [40, 41]. By comparison, there was no evidence for a nutrigenetic interaction between 5-LO promoter variants and dietary EPA/DHA in the Costa $\mathrm{Ri}$ can cohort. This finding may be due to the relatively low consumption of fish by the population in the Central Valley region of Costa Rica [42], from which this sample was collected. Nonetheless, the results from these 2 studies suggest that genetic variation in 5-LO can affect atherosclerosis in humans, particularly in the context of high dietary AA levels.

The genetic studies described above are corroborated by other positive association studies as well. For example, 2 studies recently reported association of other 5-LO variants with CVD traits in Caucasian samples $[43,44]$. In addition, DeCode Genetics reported an association between FLAP haplotypes and MI/stroke [45], which has been replicated in some populations [46-49]. Subsequently, DeCode also reported that one particular haplotype (HapK) of the LTA4H gene is associated with MI in case-control cohorts from Iceland and the USA [50]. Interestingly, the effect of HapK on MI was much stronger in African-Americans, suggesting the existence of interactions with other genes in individuals of African ancestry. It is also important to point out that such positive associations have not been uniformly observed across all studies and populations. For example, association of 5LO and FLAP variants were not associated with CVD phenotypes in several other studies [51-53], which could be due to either differences in study design, population stratification, and/or genetic heterogeneity. Alternatively, it is possible that 5 -LO pathway genes only have modest genetic contributions to CVD risk. However, it has also yet to be reported whether these other variants exhibit nutrigenetic interactions with dietary PUFAs and CVD phenotypes, analogous to the 2 studies described above for the 5-LO promoter polymorphism, which would be important studies to carry out as well.

Knowledge that 5-LO and LTs are involved in asthma previously led to the development of drugs targeting different parts of the pathway, such as zileuton, montelukast, and zafirlukast. In addition to potentially modulating LT synthesis through nutritional strategies, this raises the possibility of administering existing drugs, or newly developed ones, to target the 5-LO pathway for the prevention of CVD. However, a major question that remains to be answered is which branch of the 5-LO pathway is important for atherogenic-related processes. For example, 2 recent reports have demonstrated the efficacy of FLAP inhibitors for reducing serum inflammatory CVD risk factors in individuals carrying MI-predisposing haplotypes of the FLAP gene [54] and aortic lesion formation in mice [55]. Deficiency of the $\mathrm{LTB}_{4}$ receptors or their pharmacologic antagonism has also been reported to reduce inflammatory cytokine production in monocytes, lipid accumulation, monocyte infiltration, and smooth muscle proliferation in the artery wall, and, consequently, atherosclerosis [56-59]. Taken together with the atheroprotective phenotype of 5-LO deficient mice [28], these data suggest that $\mathrm{LTB}_{4}$ signaling, potentially through its chemotactic properties, is an important mechanism for atherogenesis.

By comparison, there is also evidence to suggest that the cysteinyl LTs have proatherogenic properties as well. In this regard, variants of the LTC4S gene have been associated with increased coronary artery calcification, carotid atherosclerosis, and risk of stroke [60-62], and montelukast, a cysteinyl LT receptor antagonist, reduces inflammatory CVD biomarkers in asthmatics [63] as well as aortic lesion formation in hyperlipidemic mouse models [64]. Thus, both branches of the 5-LO pathway appear to be proatherogenic suggesting that reduction of either type of 4-series LTs, whether through pharmacological manipulation or dietary PUFA modification (i.e. increasing EPA/DHA intake), could have beneficial effects on CVD. However, it must also be noted that genetic ablation of $5-\mathrm{LO}$, and consequently both $\mathrm{LTB}_{4}$ and the cysteinyl LTs, results in pancreatic $\beta$-cell dysfunction [33]. Thus, it will be important for future studies to address whether therapeutic strategies targeting only one branch of the 5LO pathway can provide cardioprotection while avoiding potentially undesirable metabolic adverse effects. For example, montelukast, widely used for the treatment of asthma, has potentially cardioprotective effects, as mentioned above, and has not been reported to be associated with metabolic disturbances. 


\section{Conclusions and Future Implications for Nutrigenetics of PUFAs and CVD Traits}

Until recently, reports of genes being associated with CVD traits had not been consistently confirmed in subsequent studies. In the last few years however, new evidence from genome-wide association studies has emerged suggesting that genes, which would otherwise not have been considered good candidates based on their roles in pathways unrelated to known risk factors, are conclusively associated with coronary artery disease, MI, stroke, atrial fibrillation, blood pressure, inflammatory biomarkers, and serum lipid levels [3]. Given the pleiotropic effects of EPA/DHA on a variety of cardiovascular phenotypes (table 1), it is possible that PUFAs could also exhibit nutrigenetic associations with some of the newly identified CVD genes. For example, 2 consistently observed effects of EPA/DHA are reduction of serum triglyceride levels and protection from arrhythmias. Thus, it is possible that genes controlling serum triglycerides and atrial fibrillation could interact with dietary and tissue levels of EPA/DHA to modulate these physiological effects. Such studies would be analogous to those described above for gene-dietary interactions between 5 LO variants and dietary AA and EPA/DHA. However, to detect such nutrigenetic effects will require large samples sizes where both clinical and dietary data are available.

The major challenge now facing geneticists is an understanding of the molecular and pathophysiological mechanisms through which the newly identified genes contribute to CVD traits, which is currently lacking and awaits more detailed functional experiments. In this regard, molecular tools, such as microarrays or mouse models, can be very useful for such studies, given the difficulty of dissecting complex disease genetic associations, as well as gene-gene and gene-diet interactions in humans. For example, a mouse model has been generated, which carries a transgene for the C. elegans $\omega-3$ fatty acid desaturase enzyme that converts $\omega-6$ PUFAs to $\omega-3$ PUFAs in its tissues [65]. Thus, well-controlled experiments that avoid potentially confounding factors inherent in dietary intervention studies have been carried out with these mice to study the role of EPA/DHA in a variety of disorders, including colitis [66], ocular neovascularization [67], prostate cancer [68], and acute lung injury [69]. An important study that has yet to be reported is the phenotype of these mice with respect to CVD traits, which should provide direct evidence for the effects of $\omega-3$ PUFAs on atherosclerosis in an animal model.

In summary, PUFAs, and EPA/DHA in particular, exert a variety of beneficial physiological effects that are important for the development or progression of CVD and its related traits. By modulating the levels of 5-LOpathway-derived eicosanoids through competition with AA, EPA/DHA metabolites are thought to have important anti-inflammatory properties. Biochemical, genetic, and pharmacological evidence from a number of studies in mice and humans suggests that the 5-LO/LT plays a role in CVD, particularly with respect to nutrigenetic associations involving dietary AA and EPA/DHA levels. Future studies employing a variety of complementary approaches, including novel genomics tools, animal models, and large human study populations for gene-dietary interactions, will be required to elucidate the mechanisms underlying the various effects of PUFAs on CVD.

\section{Acknowledgments}

Work in the authors' laboratories was supported by National Institute of Health grants HL079353 (H.A.), AG024154 (H.N.H.), and AT001653 (H.N.H.). N.R. was supported through a Multidisciplinary Research Training Grant in Gerontology(T32AG00037). A portion of this work was conducted in a facility constructed with support from the National Institute of Health Research Facilities Improvement Program (RR10600-01, CA62528-01, RR14514-01) from the National Center for Research Resources.

\section{References}

1 Lusis AJ: Atherosclerosis. Nature 2000;407: 233-241.

2 Lusis AJ, Weinreb A, Drake TA, Allayee H: Genetics of atherosclerosis; in Topol EJ (ed): Textbook of Cardiovascular Disease. Philadelphia, Lippincott-Raven, 2002, chapter 98.

3 National Human Genome Research Institute (NHGRI): A catalog of published genomewide association studies. www.genome.gov/ gwastudies (accessed 17 August 2009).

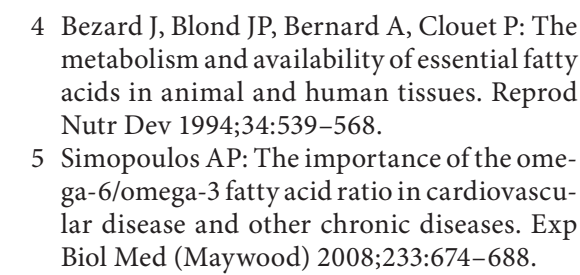

6 Nakamura MT, Nara TY: Structure, function, and dietary regulation of delta6, delta5, and delta9 desaturases. Annu Rev Nutr 2004; 24:345-376.

7 Brenna JT: Efficiency of conversion of alphalinolenic acid to long chain n-3 fatty acids in man. Curr Opin Clin Nutr Metab Care 2002; 5:127-132. 
-8 Wijendran V, Hayes KC: Dietary n-6 and n-3 fatty acid balance and cardiovascular health. Annu Rev Nutr 2004;24:597-615.

-9 Goyens PL, Spilker ME, Zock PL, Katan MB, Mensink RP: Compartmental modeling to quantify alpha-linolenic acid conversion after longer term intake of multiple tracer boluses. J Lipid Res 2005;46:1474-1483.

10 Jump DB: The biochemistry of n-3 polyunsaturated fatty acids. J Biol Chem 2002;277: 8755-8758.

11 Leaf A, Kang JX, Xiao YF: Fish oil fatty acids as cardiovascular drugs. Curr Vasc Pharmacol 2008;6:1-12.

-12 Lee JH, O’Keefe JH, Lavie CJ, Marchioli R, Harris WS: Omega-3 fatty acids for cardioprotection. Mayo Clin Proc 2008;83:324332.

-13 Harris WS, Miller M, Tighe AP, Davidson MH, Schaefer EJ: Omega-3 fatty acids and coronary heart disease risk: clinical and mechanistic perspectives. Atherosclerosis 2008;197:12-24.

14 Massaro M, Scoditti E, Carluccio MA, De Caterina R: Basic mechanisms behind the effects of n-3 fatty acids on cardiovascular disease. Prostaglandins Leukot Essent Fatty Acids 2008;79:109-115.

15 Vila L: Cyclooxygenase and 5-lipoxygenase pathways in the vessel wall: role in atherosclerosis. Med Res Rev 2004;24:399-424.

16 Mori TA, Beilin LJ: Omega-3 fatty acids and inflammation. Curr Atheroscler Rep 2004;6: 461-467.

17 Calder PC: n-3 polyunsaturated fatty acids, inflammation, and inflammatory diseases. Am J Clin Nutr 2006;83:1505S-1519S.

18 Peters-Golden M, Brock TG: Intracellular compartmentalization of leukotriene biosynthesis. Am J Respir Crit Care Med 2000; 161:S36-S40.

19 Peters-Golden M, Henderson WR Jr: Leukotrienes. N Engl J Med 2007;357:18411854.

-20 Back M, Hansson GK: Leukotriene receptors in atherosclerosis. Ann Med 2006;38:493502

-21 Radmark O, Samuelsson B: 5-lipoxygenase: regulation and possible involvement in atherosclerosis. Prostaglandins Other Lipid Mediat 2007;83:162-174.

22 Nakahata N: Thromboxane A2: physiology/ pathophysiology, cellular signal transduction and pharmacology. Pharmacol Ther 2008;118:18-35.

23 von Schacky C: Prophylaxis of atherosclerosis with marine omega-3 fatty acids. A comprehensive strategy. Ann Intern Med 1987; 107:890-899.

24 Terano T, Salmon JA, Moncada S: Biosynthesis and biological activity of leukotriene B5. Prostaglandins 1984;27:217-232.

25 Mehrabian M, Allayee H: 5-Lipoxygenase and atherosclerosis. Curr Opin Lipidol 2003; 14:447-457.
26 Tymchuk CN, Hartiala J, Patel PI, Mehrabian $\mathrm{M}$, Allayee $\mathrm{H}$ : Nonconventional genetic risk factors for cardiovascular disease. Curr Atheroscler Rep 2006;8:184-192.

27 Mehrabian M, Wong J, Wang X, et al: Genetic locus in mice that blocks development of atherosclerosis despite extreme hyperlipidemia. Circ Res 2001;89:125-130.

28 Mehrabian M, Allayee H, Wong J, et al: Identification of 5-lipoxygenase as a major gene contributing to atherosclerosis susceptibility in mice. Circ Res 2002;91:120-126.

29 Zhao L, Moos MP, Grabner R, et al: The 5lipoxygenase pathway promotes pathogenesis of hyperlipidemia-dependent aortic aneurysm. Nat Med 2004;10:966-973.

-30 Poeckel D, Zemski Berry KA, Murphy RC, Funk CD: Dual 12/15- and 5-lipoxygenase deficiency in macrophages alters arachidonic acid metabolism and attenuates peritonitis and atherosclerosis in ApoE knock-out mice. J Biol Chem 2009;284:21077-21089.

31 Cao RY, St Amand T, Grabner R, Habenicht AJ, Funk CD: Genetic and pharmacological inhibition of the 5-lipoxygenase/leukotriene pathway in atherosclerotic lesion development in ApoE deficient mice. Atherosclerosis 2009;203:395-400.

32 Mehrabian M, Allayee H, Stockton J, et al: Integrating genotypic and expression data in a segregating mouse population to identify 5-lipoxygenase as a susceptibility gene for obesity and bone traits. Nat Genet 2005;37: 1224-1233.

33 Mehrabian M, Schulthess FT, Nebohacova $\mathrm{M}$, et al: Identification of ALOX5 as a gene regulating adiposity and pancreatic function. Diabetologia 2008;51:978-988.

34 Spanbroek R, Grabner R, Lotzer K, et al: Expanding expression of the 5-lipoxygenase pathway within the arterial wall during human atherogenesis. Proc Natl Acad Sci USA 2003;100:1238-1243.

-35 Qiu H, Gabrielsen A, Agardh HE, et al: Expression of 5-lipoxygenase and leukotriene A4 hydrolase in human atherosclerotic lesions correlates with symptoms of plaque instability. Proc Natl Acad Sci USA 2006;103: 8161-8166

- 36 Sanchez-Galan E, Gomez-Hernandez A, Vidal C, et al: Leukotriene B4 enhances the activity of nuclear factor-kappaB pathway through BLT1 and BLT2 receptors in atherosclerosis. Cardiovasc Res 2009;81:216-225.

- 37 Wingrove JA, Daniels SE, Sehnert AJ, et al: Correlation of peripheral-blood gene expression with the extent of coronary artery stenosis. Circ Cardiovasc Genet 2008;1:3138

38 Dwyer JH, Allayee H, Dwyer KM, et al: Arachidonate 5-lipoxygenase promoter genotype, dietary arachidonic acid, and atherosclerosis. N Engl J Med 2004;350:29-37.

39 Allayee H, Baylin A, Hartiala J, et al: Nutrigenetic association of the 5-lipoxygenase gene with myocardial infarction. Am J Clin Nutr 2008;88:934-940.
40 Gonzalez P, Reguero JR, Lozano I, Moris C, Coto E: A functional Sp1/Egrl-tandem repeat polymorphism in the 5-lipoxygenase gene is not associated with myocardial infarction. Int J Immunogenet 2007;34:127130.

41 Maznyczka A, Braund P, Mangino M, Samani NJ: Arachidonate 5-lipoxygenase (5-LO) promoter genotype and risk of myocardial infarction: a case-control study. Atherosclerosis 2008;199:328-332.

-42 Baylin A, Kabagambe EK, Ascherio A, Spiegelman D, Campos H: Adipose tissue alphalinolenic acid and nonfatal acute myocardial infarction in Costa Rica. Circulation 2003; 107:1586-1591.

43 Carlson CS, Heagerty PJ, Nord AS, et al: TagSNP evaluation for the association of 42 inflammation loci and vascular disease: evidence of IL6, FGB, ALOX5, NFKBIA, and IL4R loci effects. Hum Genet 2007;121:6575.

44 Crosslin DR, Shah SH, Nelson SC, et al: Genetic effects in the leukotriene biosynthesis pathway and association with atherosclerosis. Hum Genet 2009; 125:217-229.

45 Helgadottir A, Manolescu A, Thorleifsson G, et al: The gene encoding 5-lipoxygenase activating protein confers risk of myocardial infarction and stroke. Nat Genet 2004;36: 233-239.

-46 Helgadottir A, Gretarsdottir S, St Clair D, et al: Association between the gene encoding 5lipoxygenase-activating protein and stroke replicated in a Scottish population. Am J Hum Genet 2005;76:505-509.

-47 Girelli D, Martinelli N, Trabetti E, et al: ALOX5AP gene variants and risk of coronary artery disease: an angiography-based study. Eur J Hum Genet 2007;15:959-966.

48 Linsel-Nitschke P, Gotz A, Medack A, et al: Genetic variation in the arachidonate 5-lipoxygenase-activating protein (ALOX5AP) is associated with myocardial infarction in the German population. Clin Sci (Lond) 2008;115:309-315.

- 49 van der Net JB, Versmissen J, Oosterveer $\mathrm{DM}$, et al: Arachidonate 5-lipoxygenase-activating protein (ALOX5AP) gene and coronary heart disease risk in familial hypercholesterolemia. Atherosclerosis 2009;203: 472-478.

-50 Helgadottir A, Manolescu A, Helgason A, et al: A variant of the gene encoding leukotriene A4 hydrolase confers ethnicity-specific risk of myocardial infarction. Nat Genet 2006;38:68-74.

-51 Zee RY, Cheng S, Hegener HH, Erlich HA, Ridker PM: Genetic variants of arachidonate 5-lipoxygenase-activating protein, and risk of incident myocardial infarction and ischemic stroke: a nested case-control approach. Stroke 2006;37:2007-2011. 
- 52 Koch W, Hoppmann P, Mueller JC, Schomig A, Kastrati A: No association of polymorphisms in the gene encoding 5-lipoxygenase-activating protein and myocardial infarction in a large central European population. Genet Med 2007;9:123-129.

- 53 Assimes TL, Knowles JW, Priest JR, et al: Common polymorphisms of ALOX5 and ALOX5AP and risk of coronary artery disease. Hum Genet 2008;123:399-408.

54 Hakonarson H, Thorvaldsson S, Helgadottir A, et al: Effects of a 5-lipoxygenase-activating protein inhibitor on biomarkers associated with risk of myocardial infarction: a randomized trial. JAMA 2005;293:22452256.

55 Jawien J, Gajda M, Rudling M, et al: Inhibition of five lipoxygenase activating protein (FLAP) by MK-886 decreases atherosclerosis in apoE/LDLR-double knockout mice. Eur J Clin Invest 2006;36:141-146.

56 Aiello RJ, Bourassa PA, Lindsey S, Weng W, Freeman A, Showell HJ: Leukotriene B4 receptor antagonism reduces monocytic foam cells in mice. Arterioscler Thromb Vasc Biol 2002;22:443-449.

57 Subbarao K, Jala VR, Mathis S, et al: Role of leukotriene $\mathrm{B} 4$ receptors in the development of atherosclerosis: potential mechanisms. Arterioscler Thromb Vasc Biol 2004;24:369375 .
58 Huang L, Zhao A, Wong F, et al: Leukotriene B4 strongly increases monocyte chemoattractant protein-1 in human monocytes. Arterioscler Thromb Vasc Biol 2004;24:17831788.

59 Heller EA, Liu E, Tager AM, et al: Inhibition of atherogenesis in BLT1-deficient mice reveals a role for LTB4 and BLT1 in smooth muscle cell recruitment. Circulation 2005; 112:578-586.

60 Iovannisci DM, Lammer EJ, Steiner L, et al: Association between a leukotriene C4 synthase gene promoter polymorphism and coronary artery calcium in young women: the Muscatine Study. Arterioscler Thromb Vasc Biol 2007;27:394-399.

61 Freiberg JJ, Dahl M, Tybjaerg-Hansen A, Grande P, Nordestgaard BG: Leukotriene C4 synthase and ischemic cardiovascular disease and obstructive pulmonary disease in 13,000 individuals. J Mol Cell Cardiol 2009; 46:579-586.

62 Bevan S, Dichgans M, Wiechmann HE, Gschwendtner A, Meitinger T, Markus HS: Genetic variation in members of the leukotriene biosynthesis pathway confer an increased risk of ischemic stroke: a replication study in two independent populations. Stroke 2008;39:1109-1114.
63 Allayee H, Hartiala J, Lee W, et al: The effect of montelukast and low-dose theophylline on cardiovascular disease risk factors in asthmatics. Chest 2007;132:868-874.

64 Jawien J, Gajda M, Wolkow P, Zuranska J, Olszanecki R, Korbut R: The effect of montelukast on atherogenesis in apoE/LDLR-double knockout mice. J Physiol Pharmacol 2008; 59:633-639.

65 Kang JX: Fat-1 transgenic mice: a new model for omega-3 research. Prostaglandins Leukot Essent Fatty Acids 2007;77:263-267.

66 Hudert CA, Weylandt KH, Lu Y, et al: Transgenic mice rich in endogenous omega-3 fatty acids are protected from colitis. Proc Natl Acad Sci USA 2006;103:11276-11281.

67 Connor KM, SanGiovanni JP, Lofqvist C, et al: Increased dietary intake of omega-3polyunsaturated fatty acids reduces pathological retinal angiogenesis. Nat Med 2007; 13:868-873.

-68 Berquin IM, Min Y, Wu R, et al: Modulation of prostate cancer genetic risk by omega-3 and omega- 6 fatty acids. J Clin Invest 2007; 117:1866-1875.

69 Mayer K, Kiessling A, Ott J, et al: Acute lung injury is reduced in fat-1 mice endogenously synthesizing $\mathrm{n}-3$ fatty acids. Am J Respir Crit Care Med 2009;179:474-483. 